\title{
On Source and Channel Codes for Multiple Inputs and Outputs: Does Multiple Description Beat Space Time? ${ }^{1}$
}

\author{
Michelle Effros $^{2} \quad$ Ralf Koetter ${ }^{3} \quad$ Andrea J. Goldsmith ${ }^{4} \quad$ Muriel Médard ${ }^{5}$
}

\author{
${ }^{2}$ Dept. of Electrical Eng., 136-93, California Institute of Technology, Pasadena, CA 91125, effros@caltech.edu \\ ${ }^{3}$ Coordinated Science Laboratory, Univ, of Illinois at Urbana-Champaign, Urbana, IL 61801, koetterouiuc.edu \\ ${ }^{4}$ Dept. of Electrical Eng., Stanford University, Stanford, CA 94305, andrea@stanford.edu \\ ${ }^{5}$ Lab. for Information and Decision Systems, Massachusetts Institute of Technology, Cambridge, MA 02139, medard@mit.ødu
}

\begin{abstract}
We compare two strategies for lossy source description across a pair of unreliable channels. In the first strategy, we use a broadcast channel code to achieve a different rate for each possible channel realization, and then use a multiresolution source code to describe the source at the resulting rates. In the second strategy, we use a channel coding strategy for two independent channels coupled with a multiple description source code. In each case, we choose the coding parameters to minimize the expected end-to-end distortion in the source reconstruction. We demonstrate that in point-to-point communication across a pair of non-ergodic channels, multiple description coding can provide substantial gains relative to mulitresolution and broadcast coding. We then investigate this comparison in a simple MIMO channel. We demonstrate the inferior performance of space time coding with multiresolution source coding and broadcast channel coding relative to multiple description codes and a time sharing channel coding strategy. These results indicate that for non-ergodic channels, the traditional definition of channel capacity does not necessarily lead to the best channel code from the perspective of end-to-end source distortion.
\end{abstract}

\section{INTRODUCTION}

We consider the problem of communication over channels subject to non-ergodic link failures. An information theoretic investigation becomes tricky in this domain since even the definition of channel capacity for channels that can fail completely and for all time is nontrivial. More precisely, consider a network comprising a single transmitter-receiver pair. Our channel model involves a pair of parallel links from the transmitter to the receiver, each of which fails with some nonzero probability. Traditional definitions place the capacity of this channel at 0 since with some nonzero probability there are no working links between the transmitter and the receiver. Alternative definitions, including the notions of outage and expected capacity introduced in [1], place the capacity value somewhat higher by allowing channel coding strategies where we permit reliable receipt of a subset of the transmitted bits. This is atypical for capacity definitions but gives increased flexibility in non-ergodic communication environments.

We approach the problem at hand with the goal of minimizing the expected distortion achieved in transmitting a continuous source across the given channel. More precisely, the source is a memoryless Gaussian with mean 0 and variance

${ }^{1}$ This work was supported in part by NSF Grant \#0325324. $\sigma^{2}$, and distortion is measured as the squared difference between the observed source sample and its reconstruction at the receiver.

We consider two communication strategies.

In one strategy, we treat the channel as a three-receiver broadcast channel. The three receivers here correspond to the decoder behaviors employed when both links succeed, when exactly one link fails, and when both links fail. We use a multiresolution source code to give a single source description that can be decoded at whatever rate the decoder receives. We choose the broadcast rates to the three receivers ${ }^{1}$ to lie on the outer boundary of the broadcast channel's capacity region and to minimize the expected distortion achieved in describing the given source across the given broadcast channel. The expectation is here taken with respect to the distribution on the rates associated with the three receivers.

In the other strategy, we use multiple description coding to send distinct descriptions over the pair of channels. We choose the multiple description code that minimizes the expected distortion with respect to the given distribution on channel failures.

For simplicity, we begin by comparing these strategies on a pair of binary links. Each link is either lossless (capacity 1) or completely absent (capacity 0 ) for all time, but which of these behaviors will occur is unknown a priori. Failures of the two links occur as independent events of probability $p$.

Since any strategy for communicating across the given pair of links may be viewed as a multiple description coding strategy, an optimal multiresolution-broadcast code cannot achieve lower end-to-end expected distortion than an optimal multiple description code. Comparison of these strategies is still of interest, though, because it lends insight into more complicated channel models where the outcome of such a comparison becomes less clear.

MIMO channels provide an interesting second example for comparing the strategies at hand. We here model the channel as a complex channel from a pair of transmit antennas to a single receive antenna. Application of an Alamouti space time code effectively yields a single channel at a communication rate that is unknown to the transmitter. Time sharing yields a pair of independent channels, each of which may fail with some nonzero probability. While space time coding achieves higher channel rates, it cannot exploit the advantages of a multiple description source code. In contrast, independent channel coding yields lower channel rates but is better matched to a multiple description source coding strategy. We therefore combine the multiresolution-broadcast strategy with the space time channel code and combine multiple description

\footnotetext{
${ }^{1}$ The receiver for two link failures always gets rate zero.
} 
coding with the time sharing channel code. In comparing the end-to-end distortion achieved by each strategy, we find that for low values of the SNR multiple description source codes with independent channel codes outperform the space time channel code with multiresolution source coding. At low values of the SNR, the data rate increase due to space time coding is insufficient to surpass the advantage of multiple description coding observed in the previous channel model.

In Section II we derive a simple (but surprisingly good) lower bound on the expected distortion in the first channel model and then derive the precise performance of the multiresolution-broadcast and multiple description strategies on this channel. Section III contains a precise characterization of the second channel model and the corresponding expected distortion results. Section IV discusses the implications of our resuits and briefly describes a few extensions of this work currently under investigation.

\section{TRANSMission STRATEGIES}

\section{A Lower Bound}

The scenario described in Section I corresponds to the transmission of two packets through two separate links, each of which may fail with probability $p$. We can obtain a simple lower bound on the achievable performance by assuming that the transmitter knows beforehand if a given channel will fail. Thus, if the transmitter knows that both channels will succeed, it can transmit at a channel rate $r=1$ on each channel which may correspond to a source coding rate $R=\lambda 2 r=\lambda 2$. The parameter $\lambda$ adapts the rate at which source symbols are produced to the channel rate. In this case a normalized distortion of $2^{-4 \lambda}$ is achievable. If it is known at the transmitter that only one packet will be available at the receiver, a normalized distortion level of $2^{-2 \lambda}$ is achievable. Thus an average normalized distortion of

$\frac{D}{\sigma^{2}}=(1-p)^{2} 2^{-4 \lambda}+2 p(1-p) 2^{-2 \lambda}+p^{2}=\left((1-p) 2^{-2 \lambda}+p\right)^{2}$

is achievable in the scenario of an informed transmitter. Clearly, we expect a significantly decreased performance for an uninformed transmitter.

\section{Multiple Description Coding}

The source coding problem of two independently available error-free channels has been solved for Gaussian sources by El Gamal and Cover and Ozarow in $[2,3]$. We want to compare their solution to an alternative approach that uses a multiresolution code in conjunction with a broadcast transmission scheme. First we restate the result of $[2,3]$ specialized to our setup. We rely on the clarification of [4] in stating that result.

Let $R_{1}$ and $R_{2}$ be the per symbol rates of a two description code. Let $D_{1}$ and $D_{2}$ be the per symbol distortions associated with receiving only the first description and only the second description, respectively. Finally, let $D_{0}$ be the per symbol distortion when both descriptions are received. Then the multipie description rate-distortion region for a Gaussian source with variance $\sigma^{2}$ is given by

$$
R_{1} \geq \frac{1}{2} \log \frac{\sigma^{2}}{D_{1}}
$$

$$
\begin{aligned}
R_{2} & \geq \frac{1}{2} \log \frac{\sigma^{2}}{D_{2}}, \\
R_{1}+R_{2} & \geq R_{0},
\end{aligned}
$$

where

$R_{0}=\left\{\begin{array}{cl}\frac{1}{2} \log \frac{\sigma^{2}}{D_{0}} & D_{0} \leq d \\ \left(\sigma^{2} / D_{0}\right) \delta_{0}^{2} & d<D_{0}<d^{\prime} \\ \frac{1}{2} \log \frac{\delta_{0}}{\delta_{0}^{2}-\left(\sqrt{\delta_{1} \delta_{2}}-\sqrt{\left(D_{1}-D_{0}\right)\left(D_{2}-D_{0}\right)}\right)^{2}} & d,^{\prime} \\ \frac{1}{2} \log \frac{\sigma^{2}}{D_{1}}+\frac{1}{2} \log \frac{\sigma^{2}}{D_{2}} & D_{0}>d d^{\prime}\end{array}\right.$

$\delta_{i}=\sigma^{2}-D_{i}, d=D_{1}+D_{2}-\sigma^{2}$, and $d^{\prime}=\left(1 / D_{1}+1 / D_{2}-\right.$ $\left.1 / \sigma^{2}\right)^{-1}$. Based on the given channel model, we are particularly interested in the case of equal rates $R_{1}$ and $R_{2}$, $R_{1}=R_{2}=R=\lambda r$, and equal distortions $D_{1}$ and $D_{2}{ }^{2}{ }^{2}$ Moreover, for our purposes it is more convenient to describe the distortion as a function of the rate.

We begin by considering the boundary points $D_{0}=d$ and $D_{0}=d^{\prime}$.

When $D_{0}=d=2 D_{1}-\sigma^{2}, R_{1}+R_{2}=(1 / 2) \log \left(\sigma^{2} / D_{0}\right)$, giving $D_{0} / \sigma^{2}=2^{-2(2 R)}$ and $D_{1} / \sigma^{2}=(1 / 2)\left(1+2^{-2(2 R)}\right)$. Since $D_{0} / \sigma^{2} \geq 2^{-2(2 R)}$ for all descriptions of rate no greater than $2 R$ and the given point minimizes $D_{1}$ subject to the constraint $D_{0}=2^{-2(2 R)}$, the point

$$
\left(\frac{D_{0}}{\sigma^{2}}, \frac{D_{1}}{\sigma^{2}}\right)=\left(2^{-2(2 R)}, \frac{1}{2}\left(1+2^{-2(2 R)}\right)\right)
$$

is the only point of interest in the region $D_{0} \leq d$.

When $D_{0}=d^{\prime}=\left(2 / D_{1}-1 / \sigma^{2}\right)^{-1}=D_{1} \sigma^{2} /\left(2 \sigma^{2}-D_{1}\right)$, we get $R=(1 / 2) \log \left(\sigma^{2} / D_{1}\right)$, giving $D_{1} / \sigma^{2}=2^{-2 R}$ and $D_{0} / \sigma^{2}=$ $2^{-2 R} /\left(2-2^{-2 R}\right)$. Since no smaller $D_{1}$ value is achievable with the given description rate $R$, the point

$$
\left(\frac{D_{0}}{\sigma^{2}}, \frac{D_{1}}{\sigma^{2}}\right)=\left(\frac{2^{-2 R}}{2-2^{-2 K}}, 2^{-2 R}\right)
$$

is the only point of interest in the region $D_{0} \geq d^{\prime}$.

In the mid-region, where $d<D_{0}<d^{\prime}$, the point of interest is $2 R=(1 / 2) \log \left[\left(\sigma^{2} / D_{0}\right)\left(\sigma^{2}-D_{0}\right)^{2} /\left(\left(\sigma^{2}-D_{0}\right)^{2}-\left(\sigma^{2}-2 D_{1}+\right.\right.\right.$ $\left.\left.\left.D_{0}\right)^{2}\right)\right]$. Letting $a=D_{0} / \sigma^{2}$ and $b=D_{1} / \sigma^{2}$, we find

$$
b=\frac{1}{2}\left[(1+a)-(1-a) \sqrt{1-\frac{2^{-2(2 R)}}{a}}\right]
$$

Thus the mid-region yields

$$
\left(\frac{D_{0}}{\sigma^{2}}, \frac{D_{1}}{\sigma^{2}}\right)=\left(a, \frac{1}{2}\left[(1+a)-(1-a) \sqrt{1-\frac{2^{-2(2 R)}}{a}}\right]\right)
$$

for all $a \in\left[2^{-2(2 R)}, 2^{-2 R} /\left(2-2^{-2 R}\right)\right]$. We summarize the above equations in the following proposition.

Proposition 1 Let an i.i.d. Gaussian source with variance $\sigma^{2}$ be described by two descriptions both of which have rate $R$. The distortions $D_{1}$ and $D_{0}$ corresponding to observations of one or both descriptions. The achievable distortion region for a fixed rate $R$ is described by:

$$
\begin{aligned}
\frac{D_{0}}{\sigma^{2}} & \geq 2^{-4 R} \\
\frac{D_{1}}{\sigma^{2}} & \geq 2^{-2 R} \\
\left(\frac{D_{0}}{\sigma^{2}}, \frac{D_{1}}{\sigma^{2}}\right) & \geq\left(a,\left(\frac{1+a}{2}-\frac{1-a}{2} \sqrt{1-\frac{2^{-4 R}}{a}}\right)\right)
\end{aligned}
$$

\footnotetext{
${ }^{2}$ Here $r=1$ is the channel transmission rate
} 


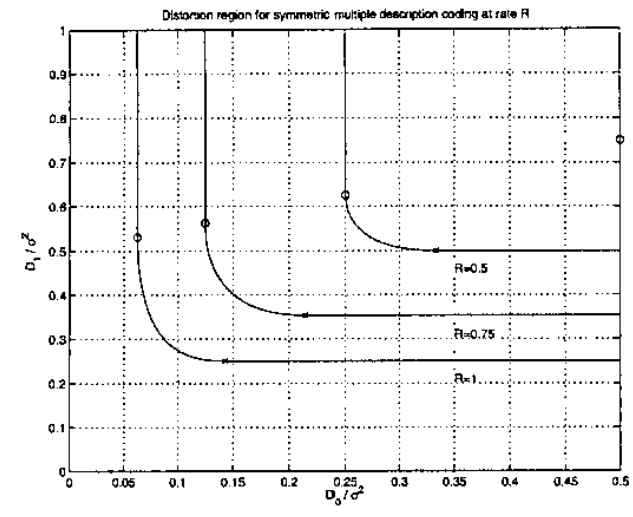

Figure 1: The expected distortion region for an i.i.d. Gaussian source with double description coding and identical description rates. $D_{1}$ is the distortion achieved by observing only one description while $D_{0}$ is the distortion achievable by observing both.

for $a \in\left[2^{-2(2 R)}, 2^{-2 R} /\left(2-2^{-2 R}\right)\right]$

Figure 1 shows the achievable distortion region for various values of $R$.

\section{The Erasure Channel Model}

\section{Separate Source and Channel Coding}

While the transmission strategy employing muitiple descriptions is, by definition, optimal for the given pair of unreliable channels, it is interesting to note how a system that uses broadcast coding performs. While multiple description coding sends two descriptions and devises mechanisms for coping with the complete loss of either description, broadcast coding guarantees reliable transmission of a fixed collection of bits to each receiver. This is accomplished by treating the original setup as the degraded broadcast erasure channel (with memory) depicted in Figure 2.

The capacity region (without common information) for the broadcast channel in Figure 2 is given by

$$
\begin{aligned}
r_{L} & =I\left(U ; Y_{L}\right)=\frac{1}{2}(1-H(b)) \\
r_{H} & =I\left(X ; Y_{H} \mid U\right)=H(b)
\end{aligned}
$$

for all $b \in[0,1 / 2]$. We here rely on the fact that the capacity for an erasure channel with the given marginal distribution is the same whether or not the channel has memory. In fact, time sharing gives a simple way to achieve this capacity. During a fraction of time equaling $r_{H}$, we transmit information to the first sink; during the remaining time $\left(1-r_{H}\right)$, we transmit information to the second sink using an erasure correcting code optimized for erasure probability $1 / 2$.

Allowing for common information gives (with $a=H(b) \in$ $[0,1])$ :

$$
\begin{aligned}
r_{L} & =\frac{1}{2}(1-a) \\
r_{L}+r_{H} & =\frac{1}{2}(1+a) .
\end{aligned}
$$

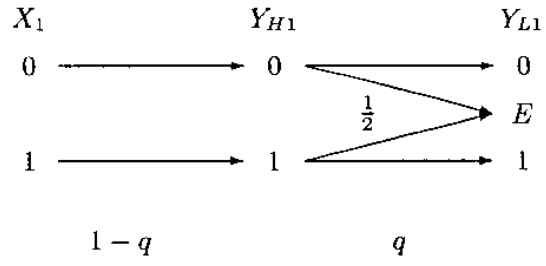

Figure 2: A single binary degraded broadcast channel.

The capacity region for a pair of independent broadcast channels simply doubles these rates.

Since the Gaussian source is successively refinable $[5,6,2]$ the distortions achieved in the first and second resolutions of a multiresolution code with incremental rates $R_{1}=\lambda 2 r_{L}$ and $R_{2}=\lambda 2 r_{H}$ (the factor of two comes from the fact that the two independent channels together give rise to twice the capacity) are $\sigma^{2} 2^{-\lambda 2(1-a)}$ and $\sigma^{2} 2^{-\lambda 2(1+a)}$, giving expected distortion over the choice of channels:

$$
{\frac{D^{2}}{\sigma}}^{2}=p^{2}+2 p(1-p) 2^{-\lambda^{2}(1-a)}+(1-p)^{2} 2^{-\lambda(1+a)} .
$$

In order to optimize this expression, notice that

$$
\frac{\partial}{\partial a}\left[\frac{D}{\sigma^{2}}\right]=\lambda(1-p) a 2^{-\lambda}\left(2 p 2^{2 \lambda a}-(1-p) 2^{-2 \lambda a}\right) .
$$

Setting this derivative equal to zero and checking boundary points gives $a \in\{1,0,(1 /(4 \lambda)) \log ((1-p) / 2 p)\}$. Since $a \in$ $[0,1]$, the latter solution is only applicable for $p \in[1 /(1+$ $\left.\left.2^{4 \lambda+1}\right), 1 / 3\right]$. Thus $D / \sigma^{2}=\min \left\{p^{2}+2 p(1-p) 2^{-2 \lambda}+(1-\right.$ $\left.p)^{2} 2^{-2 \lambda}, p^{2}+2 p(1-p)+(1-p)^{2} 2^{-4 \lambda}\right\}$ for $p \in\left[0,1 /\left(1+2^{4 \lambda+1}\right)\right] \cup$ $(1 / 3,1]$ and $D / \sigma^{2}=\min \left\{p^{2}+2 p(1-p) 2^{-2 \lambda}+(1-p)^{2} 2^{-2 \lambda}, p^{2}+\right.$ $\left.2 p(1-p)+(1-p)^{2} 2^{-4 \lambda}, p^{2}+(1-p) 2^{-2 \lambda+1} \sqrt{2 p(1-p)}\right\}$ for $p \in\left[1 /\left(1+2^{4 \lambda+1}\right), 1 / 3\right]$, giving finally

$$
\frac{D}{\sigma^{2}}=\left\{\begin{array}{cl}
p^{2}+2 p(1-p) 2^{-2 \lambda}+(1-p)^{2} 2^{-2 \lambda} & p \in\left[0, \frac{1}{1+2^{4 \lambda+1}}\right] \\
p^{2}+(1-p) 2^{-2 \lambda+1} \sqrt{2 p(1-p)} & p \in\left[\frac{1}{1+2^{4 \lambda+1}}, \frac{1}{3}\right] \\
p^{2}+2 p(1-p)+(1-p)^{2} 2^{-4 \lambda} & p \in\left[\frac{1}{3}, 1\right]
\end{array}\right.
$$

Figure 3 compares the performance of the multiresolution and multiple description strategies to the bound corresponding to an informed encoder. The multiple description code outperforms the multiresolution code for all $p \in(0,1)$.

\section{MIMIO CODING}

The findings of the previous section indicate that the scenario of non-ergodic channel behavior can open the door to significant gains in end-to-end performance. Next, we apply this approach to the area of MIMO channels. The goal of this section is not to give a self contained investigation of source and channel coding in this context. Rather, we want to illustrate the problems that arise with a specific example.

As in the simplest setup for space time coding, consider a network with two transmit antennas and a single receive antenna. Let the two complex channels (transmitter 1 to the receiver and transmitter 2 to the receiver) have complex gains $h_{1}$ and $h_{2}$. We assume a very slowly fading channel modeled by the assumption that $h_{1}$ and $h_{2}$ are time invariant (at least with respect to the amplitude of the $h_{i}$ ). We also assume that the receiver knows the $h_{i}$. While the random variables $h_{i}$ 


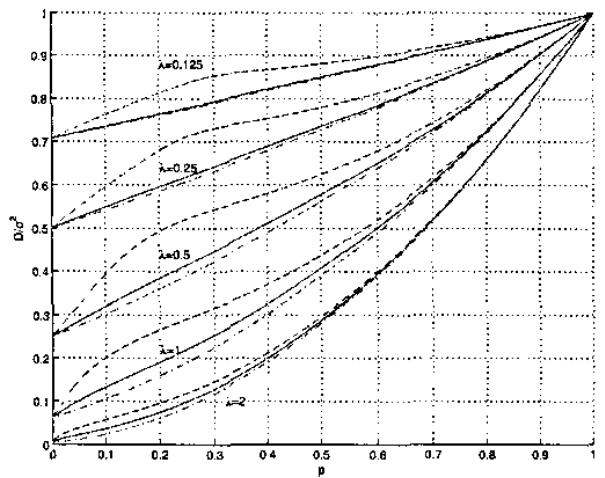

Figure 3: The normalized expected distortion $\left(D / \sigma^{2}\right)$ achieved in describing the Gaussian source across a pair of unreliable links. The dashed and solid lines show the performance of the multiresolution-broadcast coding and multiple description coding, respectively. The dash-dot line gives a lower bound on the optimal performance. We perform the comparison for $\lambda$ values ranging from 0.125 to 2 .

would typically be distributed according to some continuous distribution, we want to consider the somewhat idealized case where the amplitude of the $h_{i}$ assume only two values, namely $\{0,1\}$. The phase of the complex numbers $h_{i}$ is uniformly distributed in $[0,2 \pi]$.

Each transmit antenna transmits a complex symbol $x_{t}^{(i)}$ at time $t$. The output $y_{t}$ of the receiver antenna thus equals:

$$
y_{t}=h_{1} x_{t}^{(1)}+h_{2} x_{t}^{(2)}+n_{t}
$$

where the $n_{t}$ are independent, identically distributed complex Gaussian random variables with variance $N_{0}$.

We can use the multi-antenna channel in two different ways. Employing Strategy 1, we set $x_{t}^{(2)}$ to zero in even timeslots while $x_{t}^{(1)}$ equals zero in odd timeslots. In other words we use each transmit antenna independently of the other one in even and odd time slots, effectively creating two independent channels, one with complex gain $h_{1}$ and the other one with complex gain $h_{2}$. Each channel has capacity $\log \left(1+\left\|h_{i}\right\|^{2} \gamma\right)$ where $\gamma$ is defined as the signal to noise ratio.

In Strategy 2, we use a space time code. The natural choice for the setup described above is to choose the Alamouti scheme [7] in order to transmit information for this setup. We briefiy describe this scheme for completeness.

The idea is to transmit a complex number $x_{2 t}^{(1)}=a_{t}^{(1)}$ on the first antenna and another complex number $x_{2 t}^{(2)}=a_{t}^{(2)}$ on the second antenna. In the next time slot we choose $x_{2 t+1}^{(1)}=$ $-a_{t}^{(2) *}$ and $x_{2 t}^{(2)}=a_{t}^{(1) *}$. Two new complex numbers $a_{t+1}^{(1)}, a_{t+1}^{(2)}$ are chosen at timestep $2 t+2$. Rewriting the received signal in matrix form, we get:

$$
\left(\begin{array}{c}
y_{2 t} \\
y_{2 t+1}
\end{array}\right)=\left(\begin{array}{cc}
a_{t}^{(1)} & a_{t}^{(2)} \\
-a_{t}^{(2) *} & a_{t}^{(1) *}
\end{array}\right)\left(\begin{array}{c}
h_{1} \\
h_{2}
\end{array}\right)+\left(\begin{array}{c}
n_{2 t} \\
n_{2 t+1}
\end{array}\right) .
$$

The resuit of using the Alamouti scheme for information transmission is that we obtain two complex channels which both have the same squared complex gain $\left\|h_{1}\right\|^{2}+\left\|h_{2}\right\|^{2}$. It is interesting to note that these two channels are independent of each other, which is due to the fact that the Alamouti scheme constitutes an orthogonal design. From this observation it immediately follows that we obtain in time slots $2 t$ and $2 t+1$ two independent channels, each one with gain $\left\|h_{1}\right\|^{2}+\left\|h_{2}\right\|^{2}$ and therefore capacity $C=\log \left(1+\left(\left\|h_{1}\right\|^{2}+\left\|h_{2}\right\|^{2}\right) \gamma / 2\right)$. The term $\gamma / 2$ arises because we have to pump energy into both channels.

We see that if both $h_{1}$ and $h_{2}$ have the same amplitude $h$, then the results of both strategies are identical, i.e. we obtain a capacity of $\log \left(1+\|h\|^{2} \gamma\right)$ per multi-input channel use.

If $h_{1}$ and $h_{2}$ are different, then due to concavity of the logarithm function Strategy 1 (the time-sharing scheme) attains a smaller ergodic capacity of

$$
\frac{1}{2}\left(\log \left(1+\left\|h_{1}\right\|^{2} \gamma\right)+\log \left(1+\left\|h_{2}\right\|^{2} \gamma\right)\right)
$$

than Strategy 2

$$
\log \left(1+\left(\left\|h_{1}\right\|^{2}+\left\|h_{2}\right\|^{2}\right) \gamma / 2\right)
$$

which seems to favor space time coding over time sharing.

We emphasize that the Alamouti scheme creates independent, equally good, and reliable channels. While this is a good strategy from a channel capacity point of view, it implies that there is no room left to exploit the advantages of muitiple description coding.

On the other hand, Strategy 1, while inferior from a capacity point of view, opens the possibility of employing multiple description coding.

In order to illustrate the trade-offs involved, we next compare the expected distortion performances under the assumption that the amplitudes $\left\|h_{i}\right\|$ are drawn independently from the Bernoulli distribution with $\operatorname{Pr}\left(\left\|h_{i}\right\|=1\right)=1-p$ and $\operatorname{Pr}\left(\left\|h_{i}\right\|=0\right)=p$.

Strategy 1:

Time Sharing \& Multiple Description Coding

By Proposition 1, at rate $R=\lambda \log (1+\gamma)$ the multiple description code achieves normalized expected distortion

$$
\frac{D}{\sigma^{2}}=(1-p)^{2} a+2 p(1-p) b+p^{2}
$$

where $a \in\left[(1+\gamma)^{-4 \lambda},\{1+\gamma)^{-2 \lambda} /\left(2-(1+\gamma)^{-2 \lambda}\right)\right]$ and

$$
b=\frac{1+a}{2}-\frac{1-a}{2} \sqrt{1-\frac{(1+\gamma)^{-4 \lambda}}{a}}
$$

Numerical optimization for $a$ as a function of $p, \gamma$, and $\lambda$ yields the solid curves in Figures 4 and 5 .

Strategy 2:

\section{Space Time, Broadcast, \& Multiresolution Coding}

In this case we have a pair of channels at rates $\log (1+$ $\gamma / 2$ ) when $\left\|h_{1}\right\|=1$ and $\left\|h_{2}\right\|=0$ (or vice versa), and a pair of channels at rates $\log (1+\gamma)$ when $\left\|h_{1}\right\|=\left\|h_{2}\right\|=1$. Since the channel capacity in operation is unknown to the system encoder, we employ a broadcast code to simultaneously transmit across this pair of possible channels.

We refer to Cover and Thomas for the capacity region of the Gaussian broadcast channel. ${ }^{3}$

\footnotetext{
${ }^{3}$ The broadcast channel in question here is degraded
} 


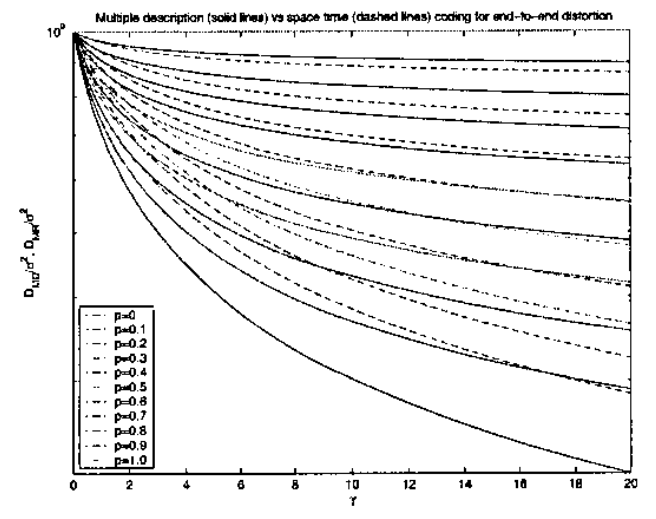

Figure 4: Space time and multiple description results for $\lambda=0.125$.

The resulting capacity region across each channel is characterized by:

$$
\begin{aligned}
r_{L}+r_{H} & \leq(\log (1+\alpha \gamma)+\log (1+(1-\alpha) /(\alpha+2 / \gamma))) \\
r_{L} & \leq(\log (1+(1-\alpha) /(\alpha+2 / \gamma)))
\end{aligned}
$$

for $\alpha \in[0,1]$. Employing a multiresolution source code at incremental rates $R_{1}=\lambda 2 r_{L}$ and $R_{2}=\lambda 2 r_{H}$ gives distortion

$$
\sigma^{2} 2^{-2\left(R_{1}+R_{2}\right)}=\sigma^{2}(1+\alpha \gamma)^{-4 \lambda}(1+(1-\alpha) /(\alpha+2 / \gamma))^{-4 \lambda}
$$

when $\left\|h_{1}\right\|=\left\|h_{2}\right\|=1$ and

$$
\sigma^{2} 2^{-2 R_{1}}=\sigma^{2}(1+(1-\alpha) /(\alpha+2 / \gamma))^{-4 \lambda}
$$

when $\left(\left\|h_{1}\right\|,\left\|h_{2}\right\|\right) \in\{(0,1),(1,0)\}$ by the successive refinabiiity of the Gaussian source. As a result, the end to end expected normalized distortion of this approach equals

$$
\begin{aligned}
{\stackrel{D^{2}}{\sigma}=}^{D^{2}} & (1-p)^{2}(1+\alpha \gamma)^{-4 \lambda}\left(1+\frac{1-\alpha}{\alpha+2 / \gamma}\right)^{-4 \lambda} \\
& +2 p(1-p)\left(1+\frac{1-\alpha}{\alpha+2 / \gamma}\right)^{-4 \lambda}+p^{2}
\end{aligned}
$$

Numerical optimization over the choice of $\alpha$ for each value of $\gamma$ and $p$ yields the dashed curves in Figure 4 and 5.

The difference between Strategy 1 (which uses multiresolution source coding and space time channel coding) and Strategy 2 (which uses time sharing and multiple description coding) for each value of $p$ appears in Figures 6 and 7 . The results demonstrate that the Alamouti scheme is a suboptimal strategy if we are interested in end-to-end distortion. ${ }^{4}$

\section{Discussion}

The results of the comparison described in Section III are illustrated in Figures 4-7. For a fixed parameter $p$ we can distinguish four regions in Figure 6 and Figure 7. At very low SNRs both schemes achieve a similar normalized distortion ${ }_{\sigma^{2}}$. very close to one since the source description rates are just too small. In particular, the difference between the two schemes becomes very small. As the SNR values increase we begin ${ }^{4}$ This holds at least for low values of $\gamma$.

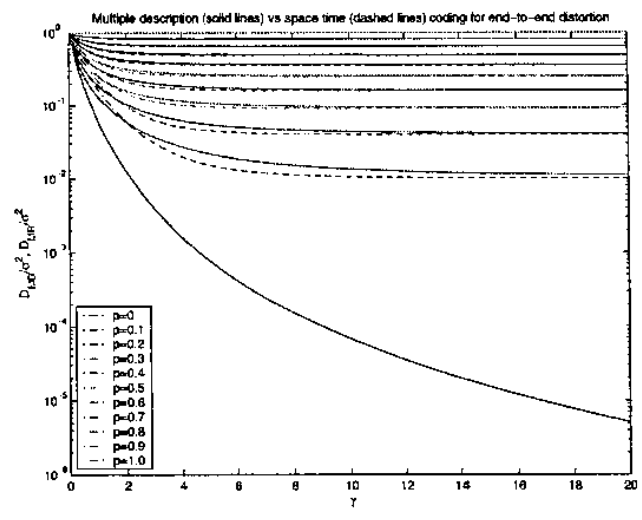

Figure 5: Space time and multiple description results for $\lambda=1$.

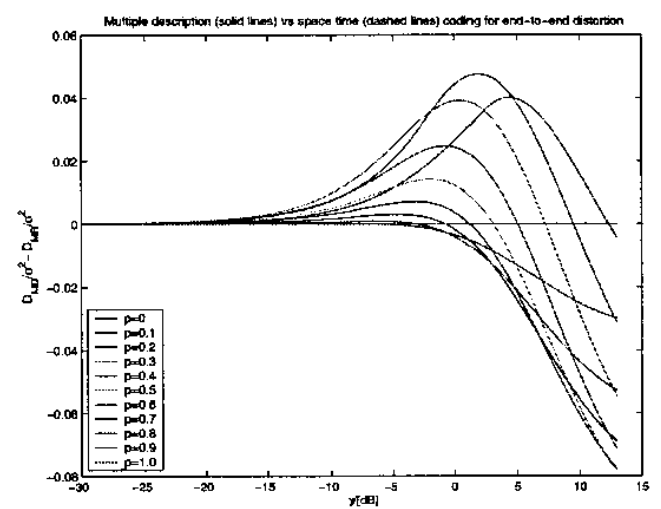

Figure 6: The difference between multiple description and space time results for $\lambda=0.125$.

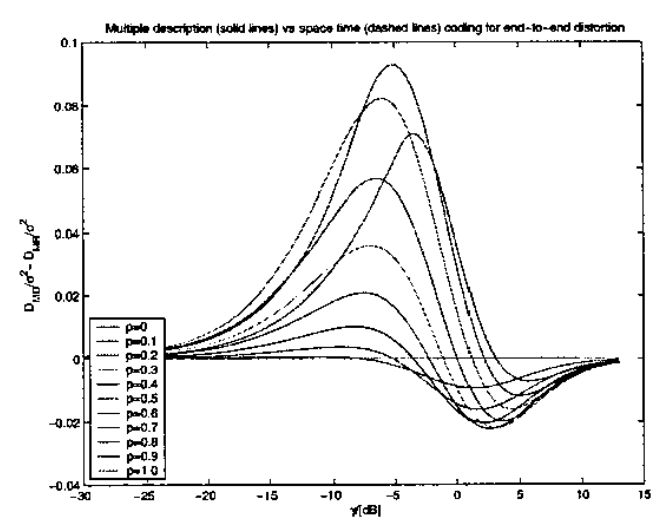

Figure 7: The difference between multiple description and space time results for $\lambda=1$. 
to observe non-vanishing description rates and the multiple description code begins to show better performance. In particular, in this range of SNR values the space time code does not yet provide the substantial gains that we would expect to see at higher SNR values. As the SNR increases the increased mutual information provided by the space time code begins to outweigh the multiple description gains of Strategy 1. Eventually, for very high SNRs the achievable distortion becomes outage determined and both schemes converge to a distortion $D=p^{2} \sigma^{2}$. Their absolute distortion difference vanishes again.

While the shown curves are specific for the Alamouti scheme, we would expect a similar behavior for general space time codes. In particular, the failure of space time codes in the low SNR regime can be interpreted in the context of existing results on MIMO capacity in the low SNR regime. Indeed, it is known that, in the low SNR regime, the capacity of a MIMO system scales as $m \mathrm{SNR}$, where $m$ is the number of receive antennas $[8,9]$, for both coherent and non-coherent channel cases. In that case, transmit antennas do not provide any added benefit from the point of view of capacity and the MIMO system acts as a single transmitter, single receiver channel. The types of channel codes that achieve capacity in that case are traditional single sender, single receiver codes operating with a single description code. The role of the receive antennas is not to acquire added refinement in information, but merely to harvest as much energy as possible. Indeed, the interference among transmit antennas, which space time codes seek to manage through orthogonality, is negligible with respect to the effect of the noise.

In this range of SNR values multiple description coding appears to be an intriguing alternative in order to capitalize on channel diversity; multiple description codes should offer an effective way to handle different non-ergodic channel realizations.

Note that the scaling with SNR and number of antennas is altogether different from the high SNR case, for which space time codes are designed. For sufficiently coherent channels, capacity is roughly $\min (m, n) \log (\mathrm{SNR})$, where $n$ is the number of transmit antennas [9]. The logarithmic dependence on SNR, as well as the dependence on both transmit and receive antennas, suggests a system which is limited by degrees of freedom rather than energy [10]. In this regime, while the multiple description code is still a good strategy, the advantages of space time coding with respect to achievable rates simply overwhelm the gains offered by the multiple description approach. It is interesting to note that the increased channel rates are due to diversity gain and as such they draw on the same resource as the multiple description codes. In particular, trading off the available diversity with respect to diversity gain and multiple description gain seems to be a very intriguing problem. Combining this problem with the design issues of multiple receive and transmit antenna codes appears to hold many challenges.

As a final thought we would like to emphasize that we believe the paper addresses a much bigger problem than multiple description coding over MIMO channels. In fact, MIMO is just a special case of exploiting non-ergodic diversity that may be offered by parallel channels. This scenario appears for example also in parallel channels through macrodiversity or soft handoff, or parailel routes through a wireless or wired network. In all of these scenarios it appears that the available diversity may be either used to stabilize a connection, thus enabling higher transmission rates, or it may be used for decreased end-to-end distortion by using a multiple description code.

\section{REFERENCES}

[1] M. Effros and A. J. Goldsmith. Capacity definitions and coding strategies for general channels with receiver side information. In Proceedings of the IEEE International Symposium on Information Theory, page 39, Cambridge, Massachusetts, August 1998. IEEE.

[2] A. A. El Gamal and T. M. Cover. Achievable rates for multiple descriptions. IEEE Transactions on Information Theory, IT28(6):851-857, November 1982.

[3] L. Ozarow. On a source-coding problem with two channels and three receivers. Bell System Technical Journal, 59:446-472, December 1980 .

[4] H. Feng and M. Effros. On the achievable region for multiple description source codes on Gaussian sources. In Proceeding. of the IEEE Intermational Symposium on Information Theory page 195, Yokohama, Japan, June 2003. IEEE.

[5] R. M. Gray and A. D. Wyner. Source coding for a simple network. Bell Systems Technical Journal, 53(9):1681-1721, November 1974.

[6] V. Koshelev. An evaluation of the average distortion for discrete schemes of sequential approximation. Probl. Pered. Inform., $17(3): 20-33,1981$

[7] S. M. Alamouti, "A simple transmitter diversity scheme for wireless communications," IEEE J. Select. Areas Commun., vol. 1, pp. 1451 1458, October 1998.

[8] D. Tse, P. Viswanath, "Fundamentals of Wireless Communications," preprint of book, http://www.eecs.berkeley.edu/ ${ }^{\sim} \mathrm{dtse} /$ main.pdf

99 L. Zheng and D. N. C. Tse, "Communication on the Grassman manifold: A geometric approach to the noncoherent multipleantenna channel," IEEE Transactions on Information Theory. Vol. 48, pp. 359-83, February 2002.

[10] M. Médard, C. Luo, "Frequency-shift keying for ultrawideband - achieving rates of the order of capacity," 40th Annual Allerton Conference on Communication, Contml, and Computing, 2002 